S1 File. Gastro-Intestinal Quality Improvement Consortium (GIQuIC) data-entry form

\begin{tabular}{|l|c|}
\hline GI Quality Improvement Consortium & GIQulC \\
\hline Colonoscopy Data Collection Form \\
\hline
\end{tabular}

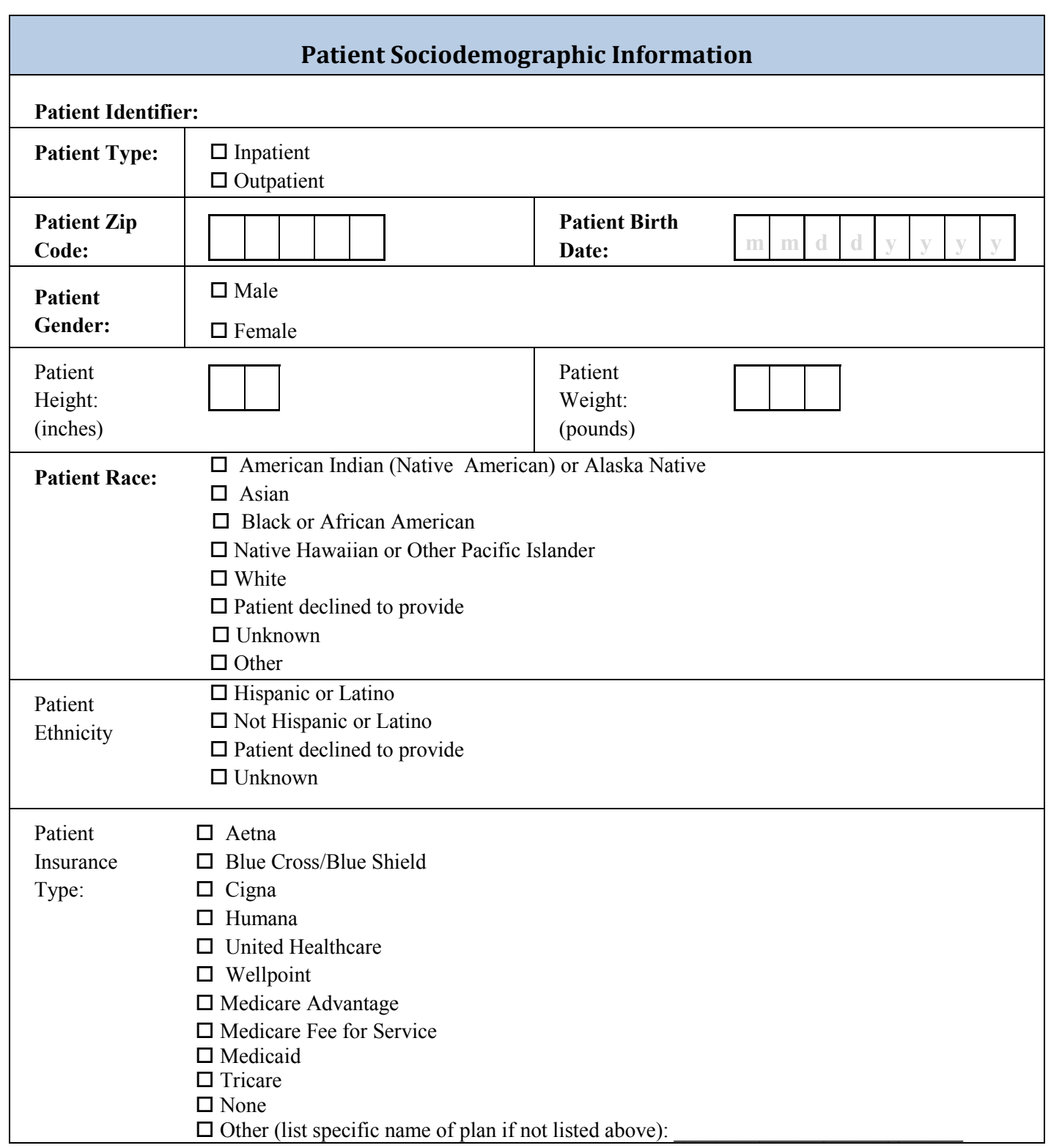

GIQUIC CRF

Version 2.1 20Aug2013

Page 1 of 7 


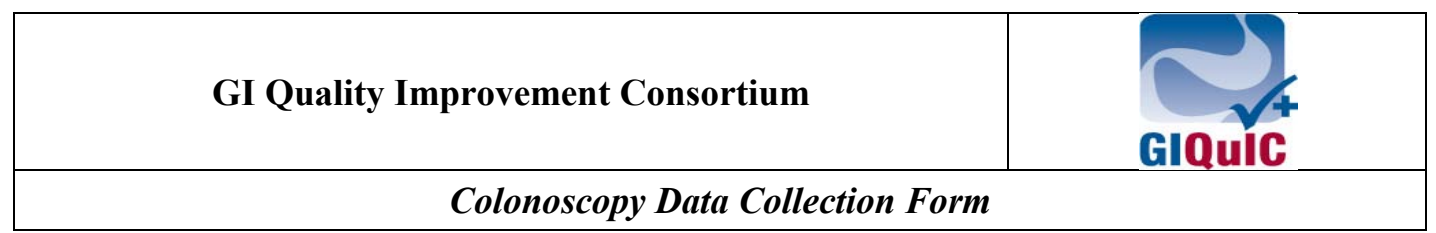

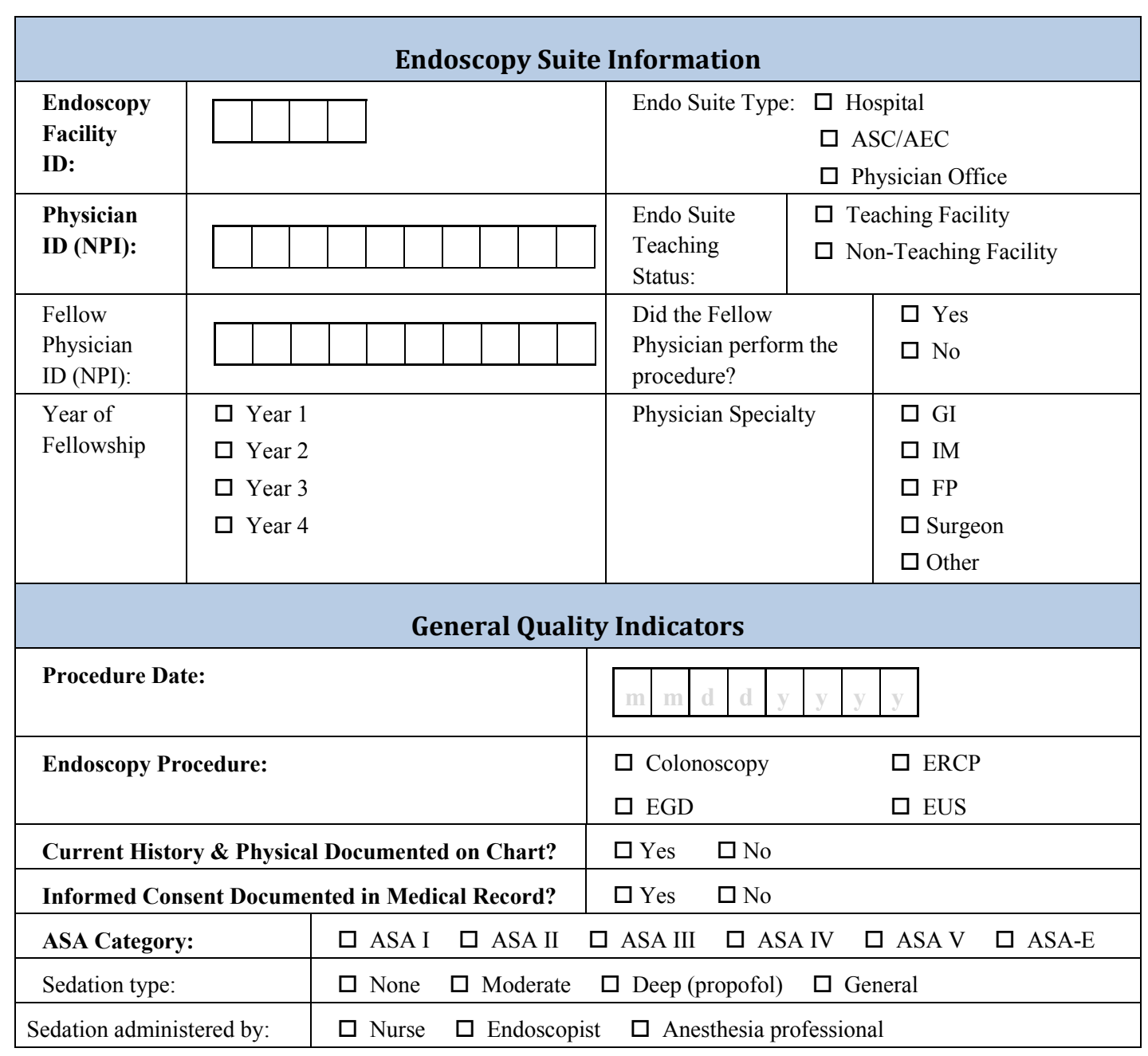

\section{Discharge Instructions}

Note: If the procedure is for an inpatient, please fill out only the questions on Diet Instructions and Medication Resumption. If the procedure is for an outpatient, please fill out all the instruction questions below.

Written Discharge Instructions provided to patient before discharge? $\quad \square$ Yes $\square$ No

Diet Instructions:

$\square$ Yes $\square$ No 


\begin{tabular}{|l|l|}
\hline GI Quality Improvement Consortium & GIQulC \\
\hline Colonoscopy Data Collection Form \\
\hline
\end{tabular}

\begin{tabular}{|l|l|}
\hline Medication Resumption / Orders Given: & $\square$ Yes $\quad$ No $\square$ N/A \\
\hline Return to Activities: & $\square$ Yes $\quad \square$ No \\
\hline Potential Delayed Complications: & $\square$ Yes $\quad \square$ No \\
\hline Medical Emergency Contact Number: & $\square$ Yes $\quad \square$ No \\
\hline \multicolumn{2}{|c|}{ Anticoagulation / Anti-platelet Therapy } \\
\hline $\begin{array}{l}\text { Anticoagulation / Anti-platelet Therapy: Patient } \\
\text { given instructions relative to resumption of therapy }\end{array}$ & $\square$ Yes $\quad \square$ No $\quad \square$ N/A \\
\hline
\end{tabular}

\begin{tabular}{|c|c|c|c|c|c|}
\hline \multicolumn{6}{|c|}{ Colonoscopy Procedure Quality Indicators } \\
\hline \multirow[t]{2}{*}{ Colonoscopy Type: } & \multicolumn{4}{|c|}{$\square$ Colon Cancer Screening $\quad \square$ Surveillance } & $\square$ Diagnostic \\
\hline & \multicolumn{2}{|l|}{$\begin{array}{l}\text { If Screening or Surveillance, } \\
\text { Year of previous colonoscopy: }\end{array}$} & \begin{tabular}{l|l|}
$\mathrm{y}$ & $\mathrm{y}$ \\
\end{tabular} & $\mathrm{y}$ & $\mathrm{y}$ \\
\hline $\begin{array}{l}\text { Bowel Prep Quality: } \\
\text { (Bowel Prep is adequate if sufficient to } \\
\text { accurately detect polyps } \geq 6 \mathrm{~mm} \text { in size; } \\
\text { Inadequate if it is NOT sufficient to } \\
\text { accurately detect polyps } \geq 6 \mathrm{~mm} \text { ) }\end{array}$ & $\square$ Adequate & quate & & & \\
\hline
\end{tabular}

\begin{tabular}{|c|l|}
\hline \multicolumn{2}{|l|}{ Colonoscopy Indication - Select at least one (1) reason for performing the colonoscopy } \\
\hline$\square$ & Evaluation of unexplained GI bleeding \\
\hline$\square$ & Unexplained iron deficiency anemia \\
\hline$\square$ & Screening for colonic neoplasia \\
\hline$\square$ & Surveillance due to prior colonic neoplasia \\
\hline$\square$ & $\begin{array}{l}\text { Inflammatory bowel disease of the intestine if more precise diagnosis or determination of the } \\
\text { extent / severity of activity of disease will influence immediate / future management }\end{array}$ \\
\hline$\square$ & Clinically significant diarrhea of unexplained origin \\
\hline$\square$ & $\begin{array}{l}\text { Evaluation of barium enema or other imaging study of an abnormality that is likely to be clinically } \\
\text { significant, such as filling defect or stricture }\end{array}$ \\
\hline$\square$ & $\begin{array}{l}\text { Intraoperative identification of a lesion not apparent/found at surgery (e.g. polypectomy site or } \\
\text { bleeding source) }\end{array}$ \\
\hline
\end{tabular}




\begin{tabular}{|l|c|}
\hline GI Quality Improvement Consortium & GIQulC \\
\hline Colonoscopy Data Collection Form \\
\hline
\end{tabular}

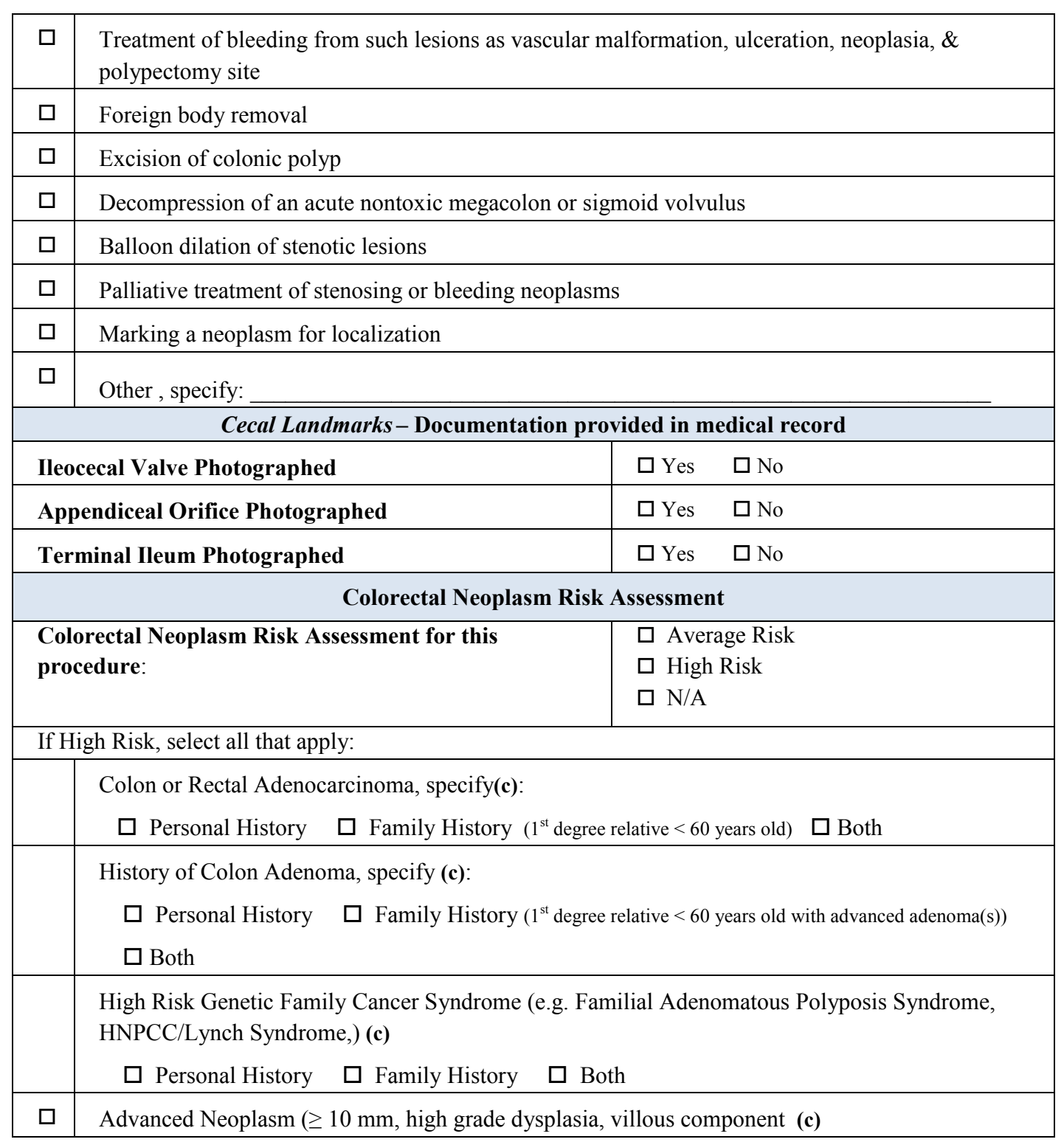




\begin{tabular}{|l|c|}
\hline GI Quality Improvement Consortium & GIQuiC \\
\hline Colonoscopy Data Collection Form \\
\hline
\end{tabular}

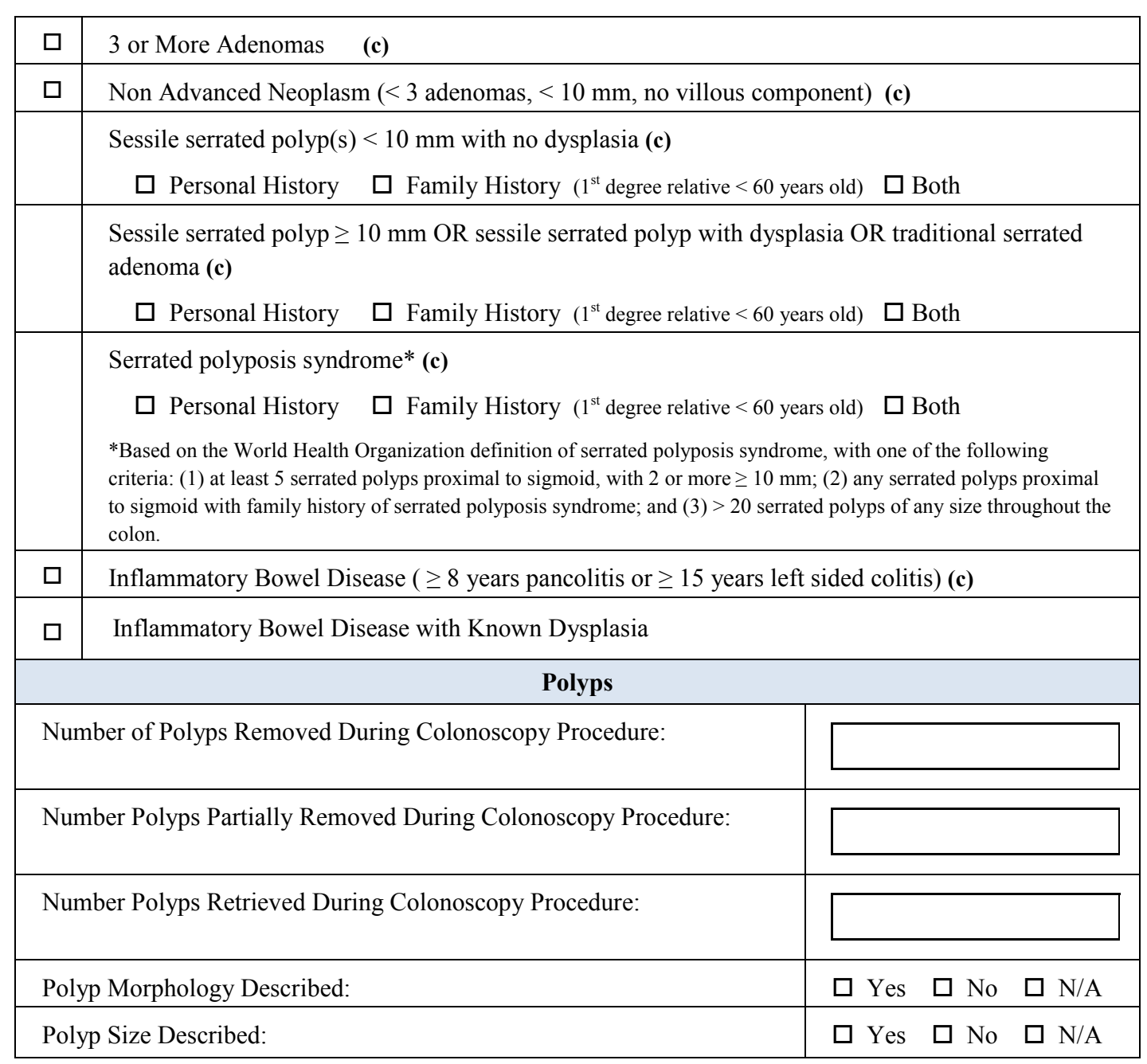




\begin{tabular}{|l|l|}
\hline GI Quality Improvement Consortium & GIQulC \\
\hline Colonoscopy Data Collection Form \\
\hline \multicolumn{2}{|c|}{} \\
\hline
\end{tabular}

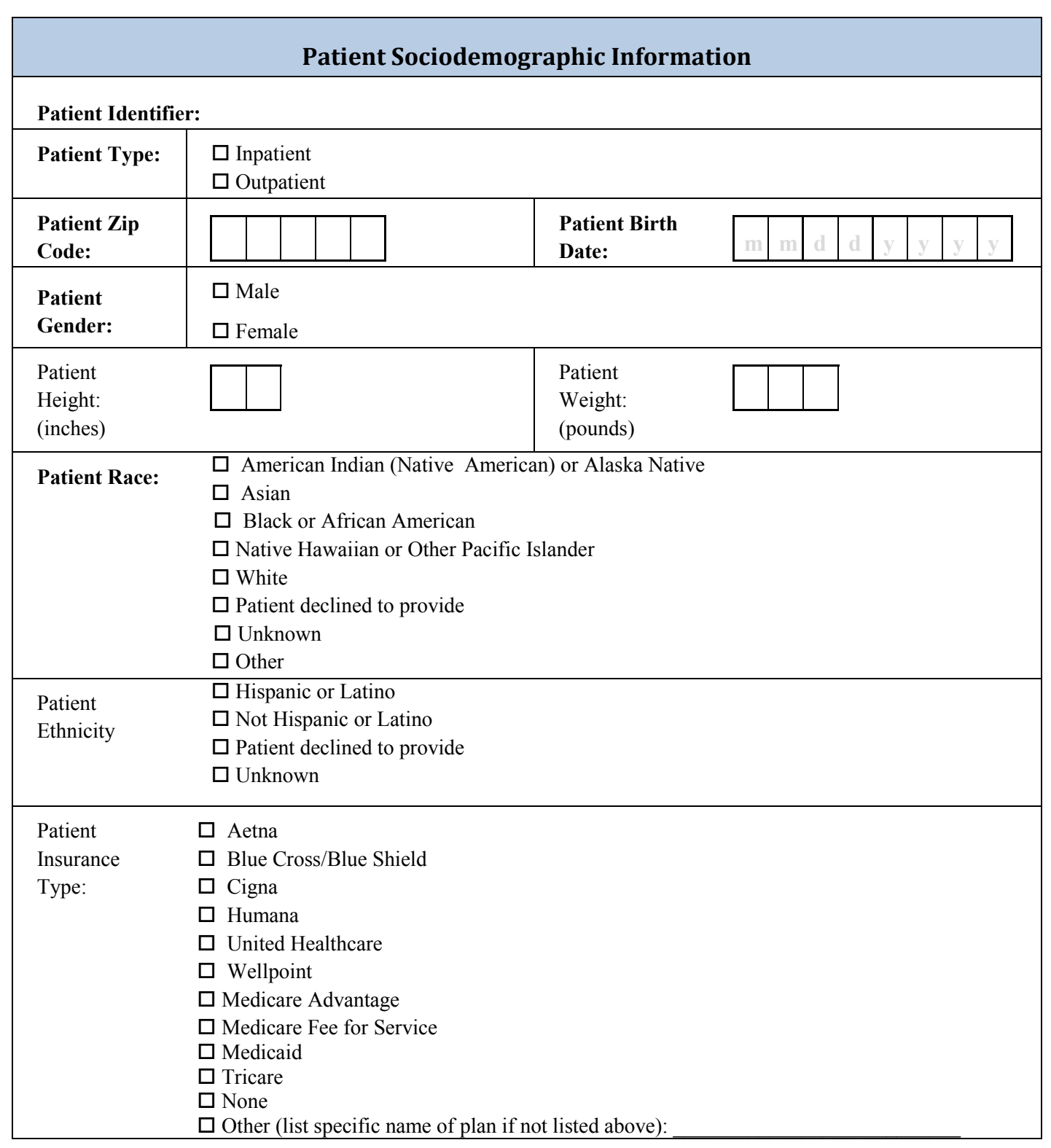




\begin{tabular}{|l|c|}
\hline GI Quality Improvement Consortium & GIQulC \\
\hline Colonoscopy Data Collection Form \\
\hline
\end{tabular}

\begin{tabular}{|l|l|}
\hline \multicolumn{2}{|c|}{ Please specify immediate adverse events(s) occurring the same day, before the patient leaves the } \\
endoscopy facility
\end{tabular}


